[4] A. Iftar, "Decentralized estimation and control with overlapping input, state and output decomposition," Automatica, vol. 29, pp. 511-516, 1993.

[5] A. Iftar and U. Özgüner, "Overlapping decompositions, expansions, contractions and stability of hybrid systems," IEEE Trans. Automat. Contr., vol. 43, pp. 1040-1055, Aug. 1998.

[6] M. Ikeda and D. D. Siljak, "Overlapping decompositions expansions and contractions of dynamic systems," Large Scale Syst., vol. 1, pp. 29-38, 1980.

[7] - "Overlapping decentralized control with input, state and output inclusion," Control Theory Adv. Technol., vol. 2, pp. 155-172, 1986.

[8] M. Ikeda, D. D. Siljak, and D. E. White, "Decentralized control with overlapping information sets," J. Optim. Theory Appl., vol. 34, pp. 279-310, 1981 .

[9] M. Ikeda, D. D. Šiljak, and D. E. White, "An inclusion principle for dynamic systems," IEEE Trans. Automat. Contr., vol. AC-29, pp. 244-249, Mar. 1984.

[10] M. Krstić, I. Kanellakopoulos, and P. Kokotović, Nonlinear and Adaptive Control Design. New York: Wiley, 1995.

[11] R. Krtolica, M. Hodžić, and D. D. Siljak, "A stochastic inclusion principle," in Differential Equations: Stability and Control, S. Elaydi, Ed. New York: Marcel Dekker, 1991, pp. 295-320.

[12] L. Padulo and M. Arbib, Systems Theory. Washington, DC: Hemisphere, 1974.

[13] G. J. Pappas, G. Laferriere, and S. Sastry, "Hierarchical consistent control systems," IEEE Trans. Automat. Contr., vol. 45, pp. 1144-1160, June 2000.

[14] M. E. Sezer and D. D. Šiljak, "Validation of reduced order models for control systems design," J. Guidance, Control Dyna., vol. 5, pp. 430-437, 1982

[15] D. D. Šiljak, Decentralized Control of Complex Systems. New York: Academic, 1991.

[16] S. S. Stanković, X. B. Chen, M. R. Mataušek, and D. D. Šiljak, "Stochastic inclusion principle applied to decentralized automatic generation control," Int. J. Control, vol. 72, pp. 276-288, 1999.

[17] S. S. Stanković, M. J. Stanojević, and D. D. Šiljak, "Decentralized overlapping control of a platoon of vehicles," IEEE Trans. Contr. Syst. Technol., vol. 8, pp. 816-832, Sept. 2000.

[18] S. S. Stanković and D. D. Šiljak, "Contractibility of overlapping decentralized control," Syst. Control Lett., vol. 44, pp. 189-200, 2001

[19] W. M. Wonham, Linear Multivariable Control: A Geometric Approach. New York: Springer-Verlag, 1979.

\section{Local Convex Directions for Hurwitz Stable Polynomials}

A. Bülent Özgüler and Karim Saadaoui

Abstract-A new condition for a polynomial $p(s)$ to be a local convex direction for a Hurwitz stable polynomial $q(s)$ is derived. The condition is in terms of polynomials associated with the even and odd parts of $p(s)$ and $q(s)$, and constitutes a generalization of Rantzer's phase-growth condition for global convex directions. It is used to determine convex directions for certain subsets of Hurwitz stable polynomials.

Index Terms-Convex directions, polynomials, robust control, stability.

\section{INTRODUCTION}

The main motivation for studying convex directions for Hurwitz stable polynomials comes from the edge theorem [2] which states

Manuscript received November 28, 2000; revised April 9, 2001 and August 20, 2001. Recommended by Associate Editor A. Datta.

The authors are with the Department of Electrical and Electronics Engineering, Bilkent University, Bilkent, Ankara 06533, Turkey (e-mail ozguler@ee.bilkent.edu.tr; karim@ee.bilkent.edu.tr).

Publisher Item Identifier S 0018-9286(02)02837-4. that, under mild conditions, it is enough to establish the stability of the edges of a polytope of polynomials in order to conclude the stability of the entire polytope. Each edge is a convex combination $\lambda r(s)+(1-\lambda) q(s), \lambda \in[0,1]$ of two vertex polynomials $r(s), q(s)$. If the difference polynomial $p(s)=r(s)-q(s)$ is a convex direction for $q(s)$, then the stability of the entire edge can be inferred from the stability of the vertex polynomials. In [15], Rantzer gave a condition which is necessary and sufficient for a given polynomial to be a convex direction for the set of all Hurwitz stable polynomials. However, this global requirement is unnecessarily restrictive when examining the stability of a particular segment of polynomials and it is of more interest to determine conditions for a polynomial to be a convex direction for a given polynomial, or still better, for specified subsets of Hurwitz stable polynomials.

Various solutions to the edge stability problem are already well known [1], [3]. Bialas [4] gave a solution in terms of the Hurwitz matrices associated with $r(s)$ and $q(s)$. The segment lemma of [6] gives another condition which requires checking the signs of two functions at some fixed points. In [9], [13], and [16], various definitions of local convex directions have been used. Among these, the following geometric characterization of [9] is the most relevant one to edge stability we have described above: A polynomial $p(s)$ is called a (local) convex direction for $q(s)$ if the set of $\alpha>0$ for which $q(s)+\alpha p(s)$ is Hurwitz stable is a single interval on the real line. Note that, if $p(s)$ is a convex direction in this sense, the stability of $q(s)$ and $p(s)+q(s)$ implies the stability of $q(s)+\alpha p(s)$ for all $\alpha \in[0,1]$, but not vice versa, i.e., the main definition used in [9] and [13] is more stringent than the one concerning the edge stability. In this note, we will use the definition given in [16], namely, a local convex direction with respect to $q(s)$ is a polynomial $p(s)$ such that all polynomials which belong to the convex combination of $q(s)$ and $q(s)+p(s)$ are Hurwitz stable. In [9], it is also shown that if one requires the local condition to be satisfied for each Hurwitz stable $q(s)$, then Rantzer's condition is obtained.

One motivation for deriving an alternative condition to those of [4] and [6] is to make contact with Ranzter's condition starting with the less stringent definition of local convexity. A second motivation is that none of the above local results seem to be suitable in determining convex directions for subsets of Hurwitz stable polynomials. Our main result in Theorem 1 is shown to be suitable for obtaining convex directions for certain subsets of Hurwitz stable polynomials. The condition provided in Theorem 1 also gives Rantzer's condition in a rather straightforward manner when it is satisfied by every Hurwitz stable polynomial. It is thus one natural local version of the global condition of Rantzer.

The note is organized as follows. In Section II, some properties of Hurwitz stable polynomials are reviewed. In Section III, we state and prove our main result, Theorem 1, which gives a new condition for checking edge stability. An application of Theorem 1 to subsets of polynomials is given in Corollary 1. Finally, in Section IV, Ranzter's condition for global convex directions is rederived based on the local condition of Theorem 1 . Some preliminary results of this paper are reported in [14].

\section{Preliminaries}

A polynomial $p(s)$ is called a global convex direction (for all Hurwitz stable polynomials of degree $n$ ) if for any Hurwitz stable polynomial $q(s)$ the implication

$$
\begin{aligned}
& q(s)+p(s) \text { is Hurwitz stable and } \operatorname{deg}(q+\lambda p)=n \\
& \quad \forall \lambda \in[0,1] \Rightarrow q(s)+\lambda p(s) \text { is Hurwitz stable } \forall \lambda \in(0,1)
\end{aligned}
$$


holds. Rantzer, in [15], has shown that a polynomial $p(s)$ is a convex direction if and only if it satisfies the phase growth condition [15], [1]

$$
\psi_{p}^{\prime}(w) \leq\left|\frac{\sin \left(2 \psi_{p}(w)\right)}{2 w}\right| \quad \forall w>0
$$

whenever $\psi_{p}(w):=\arg p(j w) \neq 0$. The condition (1) is in a sense a complement of the phase increasing property of Hurwitz stable polynomials. For a Hurwitz stable polynomial $q(s)$, the rate of change of the argument satisfies $\psi_{q}^{\prime}(w) \geq\left|\left(\sin \left(2 \psi_{q}(w)\right)\right) / 2 w\right| \forall w>0$, where the inequality is strict if $\operatorname{deg}(q) \geq 2$. This property also given in [15] seems to be known in network theory as pointed out by [5] (see also [10] for a proof based on the Hermite-Biehler Theorem, and [11] for related conditions).

Before proceeding any further, let us fix some notation. Let $\mathbf{R}$ and $\mathbf{C}$ denote the field of real and complex numbers, respectively. For a nonzero $r \in \mathbf{R}$, let $\mathcal{S}(r)$ denote the sign of $r$. Let $\mathbf{R}[s]$ denote the set of polynomials in $s$ with coefficients in $\mathbf{R}$. Let $\mathcal{H}$ denote the set of Hurwitz stable polynomials, i.e., $q(s) \in \mathcal{H} \Rightarrow \forall s \in \mathbf{C}$ such that $q(s)=0, \operatorname{Re}(s)<0$. Given $q \in \mathbf{R}[s]$, the even-odd parts ( $h(u), g(u)$ ) of $q(s)$ are the unique polynomials $h, g \in \mathbf{R}[u]$ such that $q(s)=h\left(s^{2}\right)+s g\left(s^{2}\right)$. A necessary and sufficient condition for the Hurwitz stability of $q(s)$ in terms of its even-odd parts $(h(u), g(u))$ is known as the Hermite-Biehler Theorem which is based on the following definition.

A pair of polynomials $(h(u), g(u))$ is said to be a positive pair [8] if $h(0) g(0)>0$, the roots $\left\{u_{i}\right\}$ of $h(u)$ and $\left\{v_{i}\right\}$ of $g(u)$ are real, negative, simple and with $k:=\operatorname{deg}(h)$ and $l:=\operatorname{deg}(g)$ either i) or ii) holds

i) $k=l$ and $0>u_{1}>v_{1}>u_{2}>v_{2}>\ldots>u_{k}>v_{l}$;

ii) $k=l+1$ and $0>u_{1}>v_{1}>u_{2}>v_{2}>\ldots>v_{l}>u_{k}$.

The Hermite-Biehler Theorem [8] statesthe following: a polynomial $q(s)$ with even-odd parts $(h(u), g(u))$ is Hurwitz stable if and only if $(h(u), g(u))$ is a positive pair.

The "root interlacing condition" i) and ii) can be replaced by positivity of certain polynomials of $u$. Consider the polynomials

$$
\begin{aligned}
V_{q}(u) & :=h^{\prime}(u) g(u)-h(u) g^{\prime}(u) \\
V_{s q}(u) & :=h(u) g(u)-u\left[h^{\prime}(u) g(u)-h(u) g^{\prime}(u)\right] .
\end{aligned}
$$

Lemma 1 [14]: Let $h, g \in \mathbf{R}[u]$ be coprime with $\operatorname{deg}(h)=$ $\operatorname{deg}(g) \geq 1$ or with $\operatorname{deg}(h)=\operatorname{deg}(g)+1 \geq 1$. Then, $(h, g)$ is a positive pair if and only if

i) all roots of $h$ and $g$ are real and negative;

ii) $V_{q}(u)>0 \forall u<0$;

iii) $V_{s q}(u)>0 \forall u<0$.

Finally, consider $\phi(K, u):=h(u)+K g(u)$ and $\psi(K, u):=$ $u g(u)+K h(u)$ for $K \in \mathbf{R}$. The equation $\phi(K, u)=0$ implicitly defines a function $u(K)$. The root sensitivity of $\phi(K, u)$ is defined by $K(d u / d K)$, and gives a measure of the variation in the root location of $\phi(K, u)$ with respect to percentage variations in $K$. The root sensitivities of $\phi(K, u)$ and $\psi(K, u)$, respectively, are easily computed to be

$$
S_{q}(u):=\frac{h(u) g(u)}{V_{q}(u)}, \quad S_{s q}(u):=\frac{u h(u) g(u)}{V_{s q}(u)} .
$$

\section{LOCAL CONVEX DIRECTIONS}

Our main result in this section yields a characterization of polynomials $p(s), q(s)$ which satisfy the local convexity condition (LCC)

$$
\begin{aligned}
& (L C C) q, q+p \in \mathcal{H} \text { and } \operatorname{deg}(q+\lambda p)=\operatorname{deg}(q) \\
& \qquad \lambda \in[0,1] \Rightarrow q+\lambda p \in \mathcal{H} \quad \forall \lambda \in(0,1) .
\end{aligned}
$$

Let $(h(u), g(u))$ and $(f(u), e(u))$ be the even-odd parts of $q(s)$ and $p(s)$, respectively. The following Theorem gives a test for LCC in terms of polynomials associated with the even-odd parts of $p(s)$ and the vertex polynomials $q(s), q(s)+p(s)$.

Theorem 1: Let $p(s), q(s)$ be polynomials with $n:=\operatorname{deg}(q)>1$. Then, LCC holds if and only if

$$
\begin{gathered}
V_{p}(u)<\left(\sqrt{V_{p+q}(u)}+\sqrt{V_{q}(u)}\right)^{2} \\
\forall u<0: f(u) e(u) \geq 0 \\
V_{s p}(u)<\left(\sqrt{V_{s(p+q)}(u)}+\sqrt{V_{s q}(u)}\right)^{2} \\
\forall u<0: f(u) e(u)<0 .
\end{gathered}
$$

Proof: [Only if] If $q+\lambda p \in \mathcal{H}$ for all $\lambda \in[0,1]$, then $(h+\lambda f, g+\lambda e)$ is a positive pair for all $\lambda \in[0,1]$. By lemma 1 , $V_{q+\lambda p}(u)>0$ and $V_{s(q+\lambda p)}(u)>0 \forall u<0$ and $\forall \lambda \in[0,1]$. The following identities are obtained by an easy computation:

$$
\begin{aligned}
V_{q+\lambda p}(u)= & (1-\lambda) V_{q}(u)+\lambda(\lambda-1) V_{p}(u) \\
& +\lambda V_{q+p}(u) \\
V_{s(q+\lambda p)}(u)= & (1-\lambda) V_{s q}(u)+\lambda(\lambda-1) V_{s p}(u) \\
& +\lambda V_{s(q+p)}(u) .
\end{aligned}
$$

Suppose for some $u<0$, the first condition in (3) fails. For this value of $u$

$$
\lambda:=\frac{\sqrt{V_{q}(u)}}{\sqrt{V_{q+p}(u)}+\sqrt{V_{q}(u)}} \in(0,1)
$$

achieves the value

$$
\begin{aligned}
& V_{q+\lambda p}(u) \\
& =\frac{\left[\left(\sqrt{V_{p+q}(u)}+\sqrt{V_{q}(u)}\right)^{2}-V_{p}(u)\right] \sqrt{V_{p+q}(u) V_{q}(u)}}{\left[\sqrt{V_{p+q}(u)}+\sqrt{V_{q}(u)}\right]^{2}} .
\end{aligned}
$$

By our hypothesis, the right-hand side is nonpositive which contradicts the fact that $V_{q+\lambda p}(u)>0$. Thus, the first condition in (3) must hold. Similarly, using (5), the second condition in (3) is obtained.

[If] Consider the identities

$$
\begin{aligned}
V_{q+\lambda p}(u)= & (1-\lambda)^{2} V_{q}(u)+\lambda(1-\lambda) A(u)+\lambda^{2} V_{q+p}(u) \\
V_{s(q+\lambda p)}(u)= & (1-\lambda)^{2} V_{s q}(u)+\lambda(1-\lambda) B(u) \\
& +\lambda^{2} V_{s(q+p)}(u)
\end{aligned}
$$

where $A(u):=V_{q+p}(u)+V_{q}(u)-V_{p}(u)$ and $B(u):=V_{s(q+p)}(u)+$ $V_{s q}(u)-V_{s p}(u)$. If $u<0$ is such that $A(u) \geq 0$, then as $V_{q}(u)>$ $0, V_{q+p}(u)>0$, the right hand side of (6) is positive for all $\lambda \in[0,1]$. If $u<0$ satisfies $A(u)<0$, then $A(u)-2 \sqrt{V_{p+q}(u) V_{q}(u)}=$ $\left(\sqrt{V_{q+p}(u)}-\sqrt{V_{q}(u)}\right)^{2}-V_{p}(u)<0$ and by (3)

$$
\begin{gathered}
{\left[\left(\sqrt{V_{q+p}(u)}+\sqrt{V_{q}(u)}\right)^{2}-V_{p}(u)\right]} \\
\cdot\left[\left(\sqrt{V_{q+p}(u)}-\sqrt{V_{q}(u)}\right)^{2}-V_{p}(u)\right] \\
=[A(u)]^{2}-4 V_{p+q}(u) V_{q}(u)<0
\end{gathered}
$$

for all $u \in\{u<0: f(u) e(u) \geq 0\}$ for which $A(u)<0$. But then for such $u$, the right-hand side of $(6)$ is nonzero for all $\lambda \in(0,1)$ so that it is positive for all $\lambda \in[0,1]$. This implies that

$$
V_{q+\lambda p}(u)>0 \quad \forall u \in\{u<0: f(u) e(u) \geq 0\}, \quad \forall \lambda \in[0,1] .
$$


By similar arguments, the identity (7) and the condition (3) imply that

$$
V_{s(q+\lambda p)}(u)>0 \quad \forall u \in\{u<0: f(u) e(u)<0\}, \quad \forall \lambda \in[0,1] .
$$

We now show that (8) and (9) imply $q+\lambda p \in \mathcal{H}$ for all $\lambda \in(0,1)$. Suppose for some $\lambda_{0} \in(0,1), q+\lambda_{0} p$ is not in $\mathcal{H}$. Then, as $q, q+p \in$ $\mathcal{H}$ and $\operatorname{deg}(q+\lambda p)$ is constant for $\lambda \in[0,1]$, by the continuity of the roots of $q+\lambda p$ with respect to $\lambda$, there exists $0<\lambda_{1} \leq \lambda_{2}<1$ such that $q+\lambda p \in \mathcal{H}, \quad \forall \lambda \in\left[0, \lambda_{1}\right) \cup\left(\lambda_{2}, 1\right]$ and one of the following two cases happen:

i) $q_{0}+\lambda_{1} p_{0}=0$ and $q_{0}+\lambda_{2} p_{0}=0$;

ii) $\left(q+\lambda_{1} p\right)\left(j w_{0}\right)=0$, or $\left(q+\lambda_{2} p\right)\left(j w_{1}\right)=0$;

where $w_{0} \neq 0$ or $w_{1} \neq 0$, and $q_{0}:=q(0), p_{0}:=p(0)$

i) Note that if $\lambda_{1} \neq \lambda_{2}$, then $q_{0}=0$ contradicting the fact that $q \in \mathcal{H}$. Hence, with $\lambda_{0}:=\lambda_{1}=\lambda_{2}$, we have $\lambda_{0}\left(q_{0}+p_{0}\right)+$ $\left(1-\lambda_{0}\right) q_{0}=0$ implying that $q_{0}$ and $q_{0}+p_{0}$ have different signs. Suppose $q_{0}>0$ and $q_{0}+p_{0}<0$. Since $q+\lambda p \in \mathcal{H} \quad \forall \lambda \in$ $\left[0, \lambda_{0}\right) \cup\left(\lambda_{0}, 1\right]$, it follows that $q_{0}+\lambda p_{0}>0, \quad \forall \lambda \in\left[0, \lambda_{0}\right)$ and $q_{0}+\lambda p_{0}<0, \quad \forall \lambda \in\left(\lambda_{0}, 1\right]$. Since all coefficients of a Hurwitz-stable polynomial are of the like sign, it follows that all coefficients of $q+\lambda p$ for $\lambda \in\left[0, \lambda_{0}\right)$ are positive and that all coefficients of $q+\lambda p$ for $\lambda \in\left(\lambda_{0}, 1\right]$ are negative. This implies that $q+\lambda_{0} p \equiv 0$ contradicting the hypothesis that $\operatorname{deg}(q+\lambda p)=$ $n$.

ii) Suppose without loss of generality that $u_{0}:=-w_{0}^{2}<0$. Then, we have $h\left(u_{0}\right)+\lambda_{1} f\left(u_{0}\right)=0$ and $g\left(u_{0}\right)+\lambda_{1} e\left(u_{0}\right)=0$ which contradicts either (8) or (9) depending on whether $f\left(u_{0}\right) e\left(u_{0}\right) \geq$ 0 or $f\left(u_{0}\right) e\left(u_{0}\right)<0$.

Remark 1: The following alternative statement eliminates the square roots in (3). Under the assumptions of Theorem $1, q+\lambda p \in \mathcal{H}$ for all $\lambda \in(0,1)$ if and only if

$$
\begin{aligned}
& u<0: f(u) e(u) \geq 0, A(u)<0 \Rightarrow A(u)^{2}<4 V_{p+q}(u) V_{q}(u) \\
& u<0: f(u) e(u)<0, B(u)<0 \Rightarrow B(u)^{2}<4 V_{s(p+q)}(u) V_{s q}(u)
\end{aligned}
$$

It is easy to see that given a polynomial $p(s)=f\left(s^{2}\right)+s e\left(s^{2}\right)$, it is a local convex direction for any Hurwitz-stable polynomial $q(s)=$ $h\left(s^{2}\right)+s g\left(s^{2}\right)$ whenever $(h(u), e(u))$ and $(f(u), g(u))$ form positive pairs. This follows by $A(u) \geq 0, B(u) \geq 0 \forall u<0$ and by Remark 1. In what follows, we identify other sets of Hurwitz stable polynomials for which $p(s)$ is a local convex direction. Consider the control system in Fig. 1. Given a family of plants $\mathcal{G}=\{G(s, \lambda)=$ $\left.\left(g\left(s^{2}\right)+\lambda\right) /\left(h\left(s^{2}\right)+\lambda\right): \lambda \in[0,1]\right\}$, it is easy to see that if a controller $C(s)=\left(s e\left(s^{2}\right)\right) /\left(f\left(s^{2}\right)\right)$ stabilizes $G(s, 0)$ then it stabilizes the whole family if and only if $p(s)=f\left(s^{2}\right)+s e\left(s^{2}\right)$ is a local convex direction for $q(s)=h\left(s^{2}\right) f\left(s^{2}\right)+s e\left(s^{2}\right) g\left(s^{2}\right)$. In order to get more concrete conditions using Theorem 1, we restrict $h\left(s^{2}\right)$ and $g\left(s^{2}\right)$ to be of first order. We thus consider certain subsets of polynomials obtained by adding zeros to even and/or odd part of a candidate convex direction $p(s)=f\left(s^{2}\right)+s e\left(s^{2}\right)$. Consider

$$
Q_{p}=\left\{q(s)=\left(k s^{2}+1\right) f\left(s^{2}\right)+s\left(l s^{2}+1\right) e\left(s^{2}\right): k>l \geq 0\right\}
$$

we assume here that $p(s) \in \mathcal{H}$ so that $Q_{p} \subset \mathcal{H}$ for a majority of values of $k$ and $l$. The case of $l>k \geq 0$ follows similar arguments and therefore it is omitted. In what follows, we use Theorem 1 to find conditions in terms of sensitivity functions $S_{p}(u)$ and $S_{s p}(u)$ such that $p(s)$ is a local convex direction for $Q_{p}$.

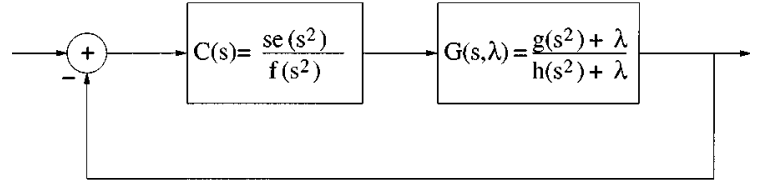

Fig. 1. A robust stabilization problem for plants of even transfer functions.

Corollary 1: Let $p(s)$ be a Hurwitz stable polynomial and $Q_{p}$ as defined in (12). The polynomial $p(s)$ is a local convex direction for $Q_{p}$ if and only if $k$ and $l$ satisfy the following conditions:

$$
\begin{aligned}
u & <0: f(u) e(u) \geq 0, \quad S_{p}(u)<\frac{2 k l u^{2}+3(k+l) u+4}{3(l-k)} \\
\Rightarrow & \frac{(l-k) u}{2 \sqrt{k l}+k+l}<S_{p}(u)<\frac{(k-l) u}{2 \sqrt{k l}-(k+l)} \\
u & <0: f(u) e(u)<0 \\
\Rightarrow & S_{s p}(u) \leq \frac{2 k l u^{2}+3(k+l) u+4}{3(k-l)} .
\end{aligned}
$$

Proof: For $q(s)=\left(k s^{2}+1\right) f\left(s^{2}\right)+s\left(l s^{2}+1\right) e\left(s^{2}\right)$ we have $A(u)=(2 k l u+3(k+l) u+4) V_{p}(u)+3(k-l) f(u) e(u)$ and $B(u)=(2 k l u+3(k+l) u+4) V_{s p}(u)-3(k-l) u f(u) e(u)$. It is lengthy but straightforward to verify that (13) is equivalent to (10). If $\forall u<0: f(u) e(u)<0, B(u)<0$ then $((k-l) u) /(2 \sqrt{k l}+k+l)<$ $S_{s p}(u)<((k-l) u) /((k+l)-2 \sqrt{k l}) \leq 0$ must be satisfied for LCC to hold. This is impossible as $S_{s p}(u)>0 \forall u<0, f(u) e(u)<0$. Condition (14) is, hence, equivalent to the following condition $\forall u<$ $0: f(u) e(u)<0 \Rightarrow B(u) \geq 0$. The result follows by Remark 1 .

Remark 2: Setting $l=0$ in Corollary 1, we get $Q_{p}=\{q(s)=$ $\left.\left(k s^{2}+1\right) f\left(s^{2}\right)+s e\left(s^{2}\right)\right\} \cdot A(u) \geq 0$ and $B(u) \geq 0$ reduce to $S_{p}(u) \geq$ $(-3 k u-4) / 3 k$ and $S_{s p} \leq(2 k u+4) / 3 k$ which can be shown to hold for every $q(s) \in \mathcal{H}$. Hence $p(s)$ is a local convex direction for all $q(s)=\left(k s^{2}+1\right) f\left(s^{2}\right)+s e\left(s^{2}\right)$ such that $q(s) \in \mathcal{H}$. This simple result is equivalent to the following robust stabilization result. Consider the family of Hurwitz-stable plants

$$
\mathcal{P}=\left\{P(s, \gamma)=\frac{f\left(s^{2}\right) s^{2}}{\gamma\left(f\left(s^{2}\right)+s e\left(s^{2}\right)\right)}: \gamma \in[1,2]\right\} .
$$

Any constant feedback gain which stabilizes the vertex plant

$$
P(s, 1)=\frac{f\left(s^{2}\right) s^{2}}{f\left(s^{2}\right)+s e\left(s^{2}\right)}
$$

also stabilizes the whole family.

\section{Convex Directions for All Hurwitz-Stable Polynomials}

In this section, we investigate the relation between the local condition of Theorem 1 and the phase growth condition of [15] which characterizes those polynomials $p(s)$ which satisfy LCC for all $q(s) \in \mathcal{H}$. In Theorem 2 below, we give an alternative proof of Rantzer's result. One part of this proof (the "if" part) is particularly straightforward and makes the connection between the local condition and the phase growth condition very clear.

The other direction of the proof requires a construction and hence it is not straightforward. We first prove a lemma used in this part of the proof of Theorem 2. The claim is that given any point $j \omega_{0}$ on the imaginary axis and any numerator polynomial $p(s)$ such that $p\left(j \omega_{0}\right) \neq$ 0 , one can design a stable denominator polynomial $\bar{r}(s)$ such that the root-locus (or the complementary root-locus) of $(p(s)) /(\bar{r}(s))$ passes through $j \omega_{0}$. 


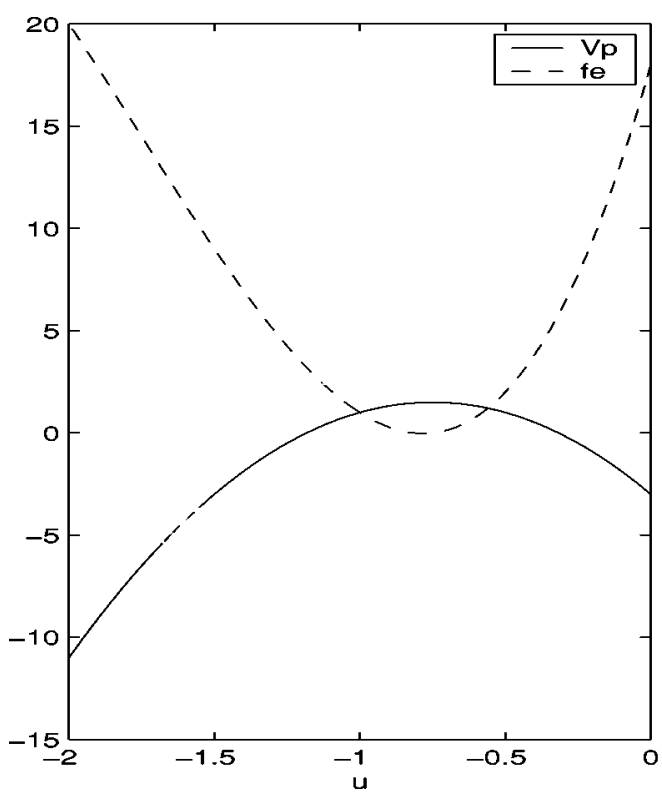

Fig. 2. Checking conditions of Theorem 2.

Lemma 2: Given a polynomial $p(s)$ with $\operatorname{deg}(p)>1$ and a real positive number $\omega_{0}$ such that $p\left(j \omega_{0}\right) \neq 0$, there exists a Hurwitz stable polynomial $\bar{r}(s)$ with $\operatorname{deg}(\bar{r}) \geq \operatorname{deg}(p)$ and a real number $\alpha$ for which $(\bar{r}+\alpha p)\left(j \omega_{0}\right)=0$.

Proof: Let $u_{0}:=-\omega_{0}^{2}$. Since $p\left(j \omega_{0}\right) \neq 0$, the polynomials $p(s), s-j \omega_{0}$ are coprime so that given any $r_{0} \in \mathbf{C}[s]$, there exists $c \in \mathbf{C}$ and $n \in \mathbf{C}[s]$ such that

$$
\left(s-j \omega_{0}\right) n(s)+p(s) c=r_{0}(s)
$$

by Euclidean algorithm in $\mathbf{C}[s]$. We can in particular choose a Hurwitz stable polynomial $r_{0}(s)$ with real coefficients such that $\operatorname{deg}\left(r_{0}\right) \geq \operatorname{deg}(p)$ and such that the even-odd components $\left(h_{0}(u), g_{0}(u)\right)$ of $r_{0}(s)$ satisfy

$$
\begin{aligned}
& \frac{g_{0}\left(u_{0}\right)}{h_{0}\left(u_{0}\right)}>\frac{f\left(u_{0}\right)}{u_{0} e\left(u_{0}\right)}>\frac{e\left(u_{0}\right)}{f\left(u_{0}\right)} \text { if } f\left(u_{0}\right) e\left(u_{0}\right)<0 \text { or } f\left(u_{0}\right)=0 \\
& \frac{h_{0}\left(u_{0}\right)}{g_{0}\left(u_{0}\right)}<\frac{u_{0} e\left(u_{0}\right)}{f\left(u_{0}\right)}<\frac{f\left(u_{0}\right)}{e\left(u_{0}\right)} \text { if } f\left(u_{0}\right) e\left(u_{0}\right)>0 \text { or } e\left(u_{0}\right)=0 .
\end{aligned}
$$

Let $c=c_{r}+j c_{i}$ for $c_{r}, c_{i} \in \mathbf{R}$ and let $n(s)=n_{r}(s)+j n_{i}(s)$ for $n_{r}, n_{i} \in \mathbf{R}[s]$. Note that $c \neq 0$ in (15), since otherwise $r_{0}(s)$ would not be Hurwitz stable. If $c_{i}=0$, then $r_{0}-c p \in \mathbf{R}[s]$ and $\bar{r}(s):=$ $r_{0}(s)$ is the desired polynomial. If $c_{i} \neq 0$, we proceed as follows. Multiplying both sides of (15) by $\left(s+j \omega_{0}\right)\left(c_{r}-j c_{i}\right)$ and equating the real and imaginary parts, we have $\left(s^{2}-u_{0}\right) m(s)-\alpha p(s)=\left(c_{i} s-\right.$ $\left.c_{r} \omega_{0}\right) r_{0}(s)=: \bar{r}(s)$ where $m(s):=c_{i} n_{r}(s)-c_{r} n_{i}(s), \alpha:=\omega_{0}\left(c_{r}^{2}+\right.$ $c_{i}^{2}$ ) and where we used the fact that $p, r_{0} \in \mathbf{R}[s]$. To complete the proof, we show that $\bar{r}(s)$ is Hurwitz stable. This requires showing that $\mathcal{S}\left(c_{r} c_{i}\right)=-1$. Evaluating (15) at $s=j \omega_{0}$, we have

$$
c_{r}+j c_{i}=\frac{r_{0}\left(j \omega_{0}\right)}{p\left(j \omega_{0}\right)}=\frac{H\left(u_{0}\right)}{F\left(u_{0}\right)}+j \omega_{0} \frac{G\left(u_{0}\right)}{F\left(u_{0}\right)}
$$

where $H(u):=h_{0}(u) f(u)-u g_{0}(u) e(u), G(u):=g_{0}(u) f(u)-$ $h_{0}(u) e(u)$ and $F(u):=f(u)^{2}-u e(u)^{2}$. Since $p\left(j \omega_{0}\right) \neq 0$ by assumption, $f(u)$ and $e(u)$ cannot be simultaneously zero at $u_{0}$. In all four possible cases i) $f\left(u_{0}\right)=0, e\left(u_{0}\right) \neq 0$, ii) $f\left(u_{0}\right) \neq 0, e\left(u_{0}\right)=$

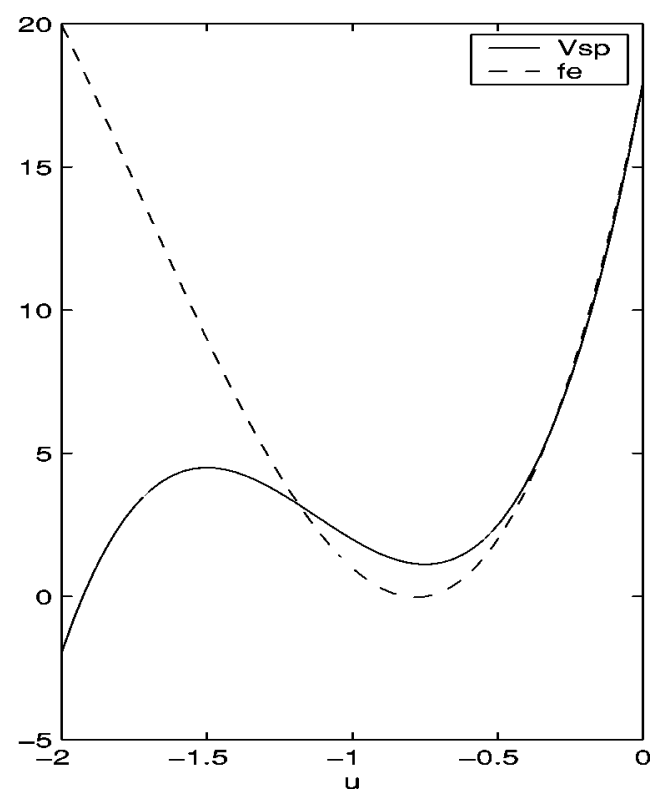

0 , iii) $\mathcal{S}\left[f\left(u_{0}\right) e\left(u_{0}\right)\right]=+1$, iv) $\mathcal{S}\left[f\left(u_{0}\right) e\left(u_{0}\right)\right]=-1$, it is straightforward to show using (16) that $\mathcal{S}\left[H\left(u_{0}\right) G\left(u_{0}\right)\right]=-1$. Since $F\left(u_{0}\right)>$ 0 , this yields that $\mathcal{S}\left(c_{r} c_{i}\right)=-1$ and the proof is complete.

In [12], Rantzer's phase-growth condition is translated into conditions on $V_{p}(u)$ and $V_{s p}(u)$. In what follows, we state the phase growth condition in this form: $p(s)$ is a global convex direction if and only if $V_{p}(u) \leq 0 \forall u<0$ such that $f(u) e(u) \geq 0$ and $V_{s p}(u) \leq 0 \forall u<0$ such that $f(u) e(u)<0$. If a given $p(s)$ need not be a convex direction for the set of all Hurwitz stable polynomials, then it is natural that the upper bounds on $V_{p}(u)$ and $V_{s p}(u)$ are relaxed. In the extreme case of a single polynomial $q(s)$, these bounds turn out to be the ones given by (3).

Theorem 2: Given a polynomial $p(s)$, the LCC holds for all $q(s) \in$ $\mathcal{H}$ if and only if

$$
\begin{array}{cc}
V_{p}(u) \leq 0 & \forall u \in\{u<0: f(u) e(u) \geq 0\} \\
V_{s p}(u) \leq 0 & \forall u \in\{u<0: f(u) e(u)<0\}
\end{array}
$$

Proof: [If] If $\operatorname{deg}(p) \leq 1$, then for $q(s)$ such that $\operatorname{deg}(q) \leq 1$, LCC is easily seen to hold. For $q(s)$ such that $\operatorname{deg}(q)>1$, if (17) holds then the conditions in (3) hold for all $q \in \mathcal{H}$ such that $q+p \in \mathcal{H}$. By Theorem 1, LCC holds for all $q \in \mathcal{H}$. If $\operatorname{deg}(p)>1$, then $\operatorname{deg}(q)>1$ in order for $\operatorname{deg}(q+\lambda p)=\operatorname{deg}(q)$ for all $\lambda \in[0,1]$. For such $q(s)$, if (17) holds, then again by Theorem $1 \mathrm{LCC}$ is satisfied.

[Only if] If $\operatorname{deg}(p) \leq 1$, then by direct computation it easy to see that (17) holds. We can therefore assume $\operatorname{deg}(p)>1$. Suppose for some $u_{0}<0$, one of the conditions in (17) fails. We construct $q \in \mathcal{H}$ for which LCC fails. Suppose that $V_{p}\left(u_{0}\right)>0$ and $f\left(u_{0}\right) e\left(u_{0}\right) \geq 0$. Note that $f\left(u_{0}\right)$ and $e\left(u_{0}\right)$ can not simultaneously be zero since otherwise $V_{p}\left(u_{0}\right)=0$. Hence, with $\omega_{0}=\sqrt{-u_{0}}$, we have $p\left(j \omega_{0}\right)=f\left(u_{0}\right)+$ $j \omega_{0} e\left(u_{0}\right) \neq 0$. By Lemma 2, there exists $\bar{r} \in \mathcal{H}, \operatorname{deg}(\bar{r}) \geq \operatorname{deg}(p)$ such that $(\bar{r}+\alpha p)\left(j \omega_{0}\right)=0$ for some $\alpha \in \mathbf{R}$. Since $\bar{r}(s)$ is Hurwitz stable , $\alpha \neq 0$. If we let $(\bar{k}(u), \bar{l}(u))$ be the even-odd components of $\bar{r}(s)$, then by $(\bar{r}+\alpha p)\left(j \omega_{0}\right)=0$ and $\alpha \neq 0$, we have

$$
(\bar{k} e-\bar{l} f)\left(u_{0}\right)=0 .
$$

Let

$$
r(s):=-\lambda_{0} p(s)+\left(s^{2}+\omega_{0}^{2}\right) \bar{r}(s)
$$




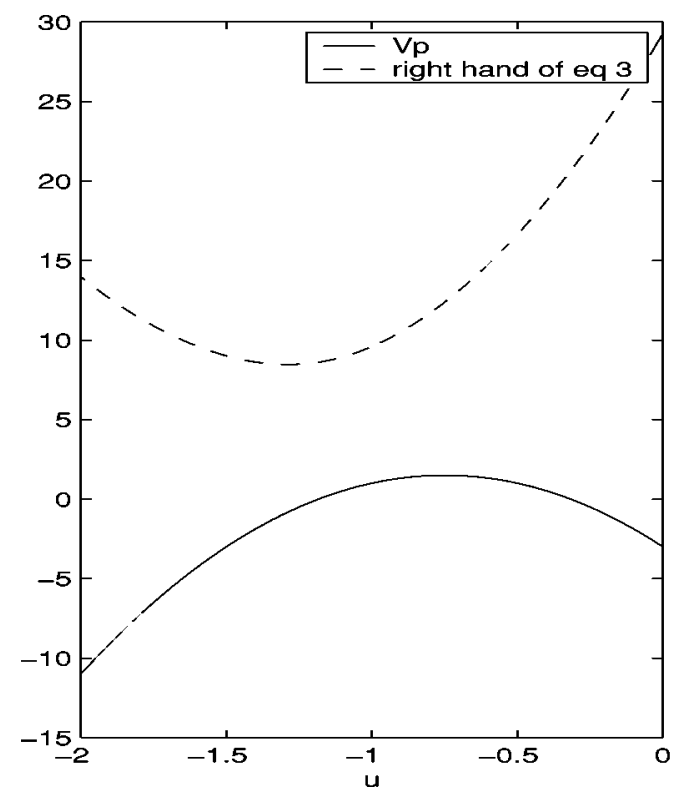

Fig. 3. Checking conditions of Theorem 1.

for some arbitrary but fixed $\lambda_{0} \in(0,1)$. If we let $(k(u), l(u))$ be the even-odd components of $r(s)$, we have $\left(k+\lambda_{0} f\right)\left(u_{0}\right)=0$ and $\left(l+\lambda_{0} e\right)\left(u_{0}\right)=0$ so that

$$
V_{r+\lambda_{0} p}\left(u_{0}\right)=0, V_{s\left(r+\lambda_{0} p\right)}\left(u_{0}\right)=0 .
$$

We now show that, there exists $\epsilon>0$ such that $V_{r+\lambda p}(u)>$ $0, V_{s(r+\lambda p)}(u)>0 \forall \lambda \in\left[\lambda_{0}-\epsilon, \lambda_{0}\right) \cup\left(\lambda_{0}, \lambda_{0}+\epsilon\right], \forall u<0$. Note that

$$
\begin{aligned}
V_{r+\lambda p}(u)= & (k+\lambda f)^{\prime}(u)(l+\lambda e)(u) \\
& -(k+\lambda f)(u)(l+\lambda e)^{\prime}(u) \\
V_{s(r+\lambda p)}(u)= & (k+\lambda f)(u)(l+\lambda e)(u)-u V_{r+\lambda p}(u) .
\end{aligned}
$$

By (19), $(k+\lambda f)(u)=\left(u-u_{0}\right) \bar{k}(u)$ and $(l+\lambda e)(u)=\left(u-u_{0}\right) \bar{l}(u)$ so that

$$
\begin{aligned}
V_{r+\lambda p}(u)= & V_{r+\lambda_{0} p}(u)+\left(\lambda-\lambda_{0}\right)(\bar{k} e-\bar{l} f)(u)+\left(\lambda-\lambda_{0}\right) \\
& \left(\bar{k}^{\prime} e-\bar{k} e^{\prime}-\bar{l}^{\prime} f+\bar{l} f^{\prime}\right)\left(u-u_{0}\right)+\left(\lambda-\lambda_{0}\right)^{2} V_{p}(u) .
\end{aligned}
$$

Hence, using (18) and (19), we have $V_{r+\lambda_{p}}\left(u_{0}\right)=\left(\lambda-\lambda_{0}\right)^{2} V_{p}\left(u_{0}\right)$ and $V_{r+\lambda_{0} p}(u)=\left(u-u_{0}\right)^{2} V_{\bar{r}}(u)$. Similarly, $V_{s(r+\lambda p)}\left(u_{0}\right)=(\lambda-$ $\left.\lambda_{0}\right)^{2} V_{s p}\left(u_{0}\right)$ and $V_{s\left(r+\lambda_{0} p\right)}(u)=\left(u-u_{0}\right)^{2} V_{s \bar{r}}(u)$. Since $\bar{r} \in \mathcal{H}$ and $\operatorname{deg}(\bar{r}) \geq 2$, we can apply Lemma 1 to obtain $V_{\bar{r}}(u)>0$ and $V_{s \bar{r}}(u)>0$ for all $u<0$. Hence, $V_{r+\lambda_{0} p}(u)>0$ and $V_{s\left(r+\lambda_{0} p\right)}(u)>$ 0 for all $u$ such that $0>u \neq u_{0}$. By our assumption, $V_{p}\left(u_{0}\right)>0$ and $f\left(u_{0}\right) e\left(u_{0}\right) \geq 0$. Hence, $V_{s p}\left(u_{0}\right)=f\left(u_{0}\right) e\left(u_{0}\right)-u_{0} V_{p}\left(u_{0}\right)>0$. Consequently, $V_{r+\lambda p}\left(u_{0}\right)>0$ and $V_{s(r+\lambda p)}\left(u_{0}\right)>0$ for all $\lambda \in$ $[0,1]$ such that $\lambda \neq \lambda_{0}$. It follows that, for some sufficiently small $\epsilon_{1}>0$, we have $V_{r+\lambda p}(u)>0$ and $V_{s(r+\lambda p)}(u)>0 \forall u<0 \forall \lambda \epsilon$ $\left[\lambda_{0}-\epsilon_{1}, \lambda_{0}\right) \cup\left(\lambda_{0}, \lambda_{0}+\epsilon_{1}\right]$. We now note, by $\left(k+\lambda_{0} f\right)(u)=$ $\left(u-u_{0}\right) \bar{k}(u),\left(l+\lambda_{0} e\right)(u)=\left(u-u_{0}\right) \bar{l}(u)$, and the fact that $(\bar{k}, \bar{l})$ is a positive pair, that all the roots of $k+\lambda f$ and $l+\lambda e$ are real and negative for all $\lambda \in\left[\lambda_{0}-\epsilon_{2}, \lambda_{0}+\epsilon_{2}\right]$ for some sufficiently small $\epsilon_{2}$. Therefore, for all $\lambda \in\left[\lambda_{0}-\epsilon, \lambda_{0}\right) \cup\left(\lambda_{0}, \lambda_{0}+\epsilon\right]$ with $\epsilon:=\min \left\{\epsilon_{1}, \epsilon_{2}\right\}$, we have that $(k+\lambda f, l+\lambda e)$ is a positive pair by Lemma 1 so that $r+$ $\left(\lambda_{0}+\epsilon\right) p, r+\left(\lambda_{0}-\epsilon\right) p \in \mathcal{H}$. If we now define $q(s):=1 /(2 \epsilon)[r(s)+$ $\left.\left(\lambda_{0}-\epsilon\right) p(s)\right]$, then $q, q+p \in \mathcal{H}, \operatorname{deg}(q+\lambda p)=\operatorname{deg}(q) \forall \lambda \in[0,1]$,

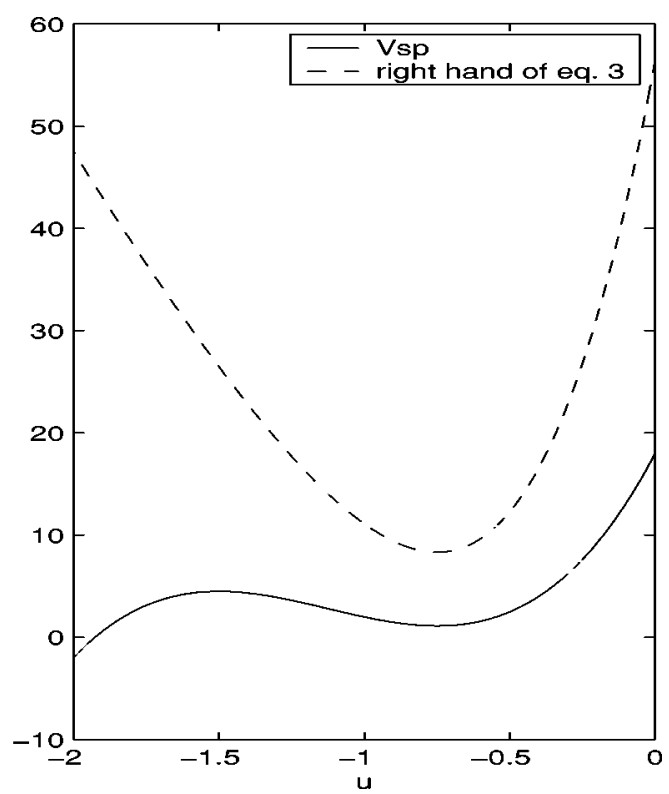

but $(q+0.5 p)\left(j \omega_{0}\right)=(1 / 2 \epsilon)\left(r+\lambda_{0} p\right)\left(j \omega_{0}\right)=0$ and LCC fails for this $q(s)$. If $u_{0}<0$ is such that $V_{s p}\left(u_{0}\right)>0$ and $f\left(u_{0}\right) e\left(u_{0}\right)<0$, then $u_{0} V_{p}\left(u_{0}\right)=f\left(u_{0}\right) e\left(u_{0}\right)-V_{s p}\left(u_{0}\right)<0$ so that $V_{p}\left(u_{0}\right)>0$. The construction of $q(s)$ for which LCC fails is exactly the same as above.

Example 1: Consider $p(s)=2 s^{5}+9 s^{3}+4 s^{2}+6 s+3$ and $q(s)=$ $0.4 s^{5}+2.1 s^{4}+1.9 s^{3}+4.2 s^{2}+1.6 s+1.6$. We can easily check that $q(s)$ and $p(s)+q(s)$ are Hurwitz stable. For $u<-2, V_{p}(u)<0$ and $V_{s p}(u)<0$. From Fig. 2 we can see that the first and second condition of Theorem 2 fail in the intervals $[-1.18,-0.81] \cup[-0.75,-0.32]$ and $[-0.81,-0.75]$, respectively. Hence $p(s)$ is not a global convex direction. On the other hand, from Fig. 3, we can see that the conditions of Theorem 1 are satisfied in the whole interval $[-2,0]$. Hence, LCC holds for the pair $(p, q)$.

\section{CONCLUSION}

Theorem 1 gives a necessary and sufficient condition for a polynomial $p(s)$ to be a local convex direction for a Hurwitz stable polynomial $q(s)$. This condition is a generalization of Rantzer's phase growth condition for global convex directions. The main advantage of the condition is that it can be used to identify "subsets" of Hurwitz stable polynomials for which a given polynomial is a convex direction. Computationally, the tests provided by [4] and [6] may be more advantageous. However, we note that the main condition for local convexity is equivalent to the positivity of polynomials (6) and (7) for $u<0$ and $\lambda \in[0,1]$. By the main result in [7], the positivity of these polynomials can be checked by performing only a finite number of elementary operations (arithmetic operations, logical operations, and sign tests).

\section{REFERENCES}

[1] B. R. Barmish, New Tools for Robustness of Linear Systems. New York: MacMillan, 1994.

[2] A. C. Bartlett, C. V. Hollot, and L. Huang, "Root location of an entire polytope of polynomials: It suffices to check the edges," Math. Control, Signals, Syst., vol. 1, pp. 61-71, 1988.

[3] S. P. Bhattacharyya, H. Chapellat, and L. H. Keel, Robust Control: The Parametric Approach. Upper Saddle River, NJ: Prentice-Hall, 1995.

[4] S. Bialas, "A necessary and sufficient condition for the stability of convex combinations of stable polynomials and matrices," Bulletin Polish Acad. Sci., vol. 33, pp. 473-480, 1985. 
[5] N. K. Bose, "Argument conditions for Hurwitz and Schur polynomials from network theory," IEEE Trans. Automat. Contr., vol. 39, pp. 345-346, Feb. 1994.

[6] H. Chapellat and S. P. Bhattacharyya, "An alternative proof of Kharitonov's theorem," IEEE Trans Automat. Contr., vol. 34, pp. 448-450, Apr. 1989.

[7] L. Xie and M. Fu, "Finite test of robust strict positive realness," Int. J. Control, vol. 64, no. 5, pp. 887-897, 1996.

[8] F. R. Gantmacher, The Theory of Matrices, Vol. II. New York: Chelsea, 1959.

[9] D. Hinrichsen and V. L. Kharitonov, "On convex directions for stable polynomials," Automat. Remote Control, vol. 58, no. 3, pp. 394-402, 1997.

[10] L. H. Keel and S. P. Bhattacharyya, "Phase properties of Hurwitz polynomials," IEEE Trans Automat. Contr., vol. 41, pp. 733-734, May 1996.

[11] M. Mansour, "Robust stability in systems described by rational functions," in Control and Dynamic Systems, C. T. Leondes, Ed. New York: Academic, 1992, vol. 51, pp. 79-128.

[12] A. B. Özgüler, "Constructing convex directions for stable polynomials," IEEE Trans. Automat. Contr., vol. 45, pp. 1569-1574, Aug. 2000.

[13] A. B. Özgüler and A. A. Koçan, "An analytic determination of stabilizing feedback gains," Institut für Dynamische Systeme, Universität Bremen, Rep. no. 321, 1994

[14] A. B. Özgüler and K. Saadaoui, "Local convex directions," in Proc. European Control Conf., Porto, Portugal, 2001, pp. 708-712.

[15] A. Rantzer, "Stability conditions for polytopes of polynomials," IEEE Trans. Automat. Contr., vol. 37, pp. 79-89, Jan. 1992.

[16] E. Zeheb, "On the characterization and formation of local convex directions for Hurwitz stability," in Stability Theory, Hurwitz Centenary Conference, R. Jeltsch and M. Mansour, Eds. Boston, MA: Birkhäuser, 1995, pp. 173-180

\section{Stable Inversion of Continuous-Time Nonlinear Systems by Finite-Difference Methods}

\author{
David G. Taylor and Song Li
}

\begin{abstract}
This note introduces finite-difference methods for stable inversion of continuous-time nonlinear systems. A relationship between the new finite-difference methods and the existing Picard methods is established. A damped Newton finite-difference method is shown to possess superior convergence properties, and its effectiveness is illustrated with an inverted pendulum example.
\end{abstract}

Index Terms-Boundary-value problems, nonminimum-phase systems, output tracking, stable inversion.

\section{INTRODUCTION}

Inversion is the process of computing reference trajectories for the plant input and state variables that are consistent with exact tracking of some given reference trajectory for the plant output. Stable inversion [2], [5] insists that the computed reference trajectories be bounded,

Manuscript received September 25, 2000; revised August 7, 2001. Recommended by Associate Editor P. Tomei. This work was supported in part by the Office of Naval Research under Grant N00014-96-1-0926, and in part by Siemens AG.

D. G. Taylor is with Georgia Institute of Technology, School of Electrical and Computer Engineering, Atlanta, GA 30332-0250 USA (david.taylor@ece.gatech.edu).

$\mathrm{S}$. Li is with Ciena Corporation, Department of Operations Engineering, Linthicum, MD 21090 USA (e-mail: SoLi@ ciena.com).

Publisher Item Identifier S 0018-9286(02)02838-6. whereas classical inversion [8] forces the computed reference trajectories to be causal. Stable inversion reduces to classical inversion for the special case of minimum-phase systems. For the general case of nonminimum-phase systems, the existing numerical methods for stable inversion are based on a Picard process that requires decoupling coordinate transformations to separate the stable and unstable parts of the inverse system [5], [9], [6], [7], [14]. The Picard iteration is implemented using a combination of forward-time and backward-time numerical integration or, if desired, by discrete Fourier transform techniques.

In this note, a new class of numerical methods for stable inversion is proposed. The new methods are motivated from the interpretation of stable inversion as a standard type of two-point boundary-value problem [2]. From among the vast array of numerical methods available for such problems [12], [1], the finite-difference methods (sometimes called relaxation methods [13]) seem most suitable for stable inversion. The application of a finite-difference method consists of three steps: 1) choose mesh points on a time interval to define where approximate solution values are sought; 2) form a set of algebraic equations for the approximate solution values by replacing derivatives with difference quotients; 3) solve the resulting system of simultaneous equations to obtain the approximate solution values. Interpolation may then be used if approximate solution values are needed between mesh points. If the iteration process used in the third step is based on a damped Newton scheme, the resulting finite-difference method outperforms existing Picard methods by enlarging the region of convergence and increasing the local rate of convergence from linear to quadratic.

An inverted pendulum example is included to illustrate the stable inversion process. A damped Newton finite-difference method and the traditional Picard method are both implemented. The numerical results reveal the significant practical advantages of the damped Newton finitedifference method.

\section{Two-Point Boundary-VALUe Problem}

Consider a nonlinear system defined by

$$
\begin{aligned}
& \dot{x}=f(x)+g(x) u \\
& y=h(x)
\end{aligned}
$$

where $x \in R^{n}, u \in R, y \in R$, and $f(x), g(x)$ and $h(x)$ are smooth functions with $f(0)=0$ and $h(0)=0$. The following analysis is local to the equilibrium point at $(u, x, y)=(0,0,0)$.

It is well known [11], [14] that if this system has relative degree $r$ at $x=0$, then there exists a local change of coordinates $(\xi, \eta)=\Phi(x)$ with $(0,0)=\Phi(0)$ such that the system appears in the normal form

$$
\begin{aligned}
\dot{\xi}_{i} & =\xi_{i+1}, \quad i=1, \ldots, r-1 \\
\dot{\xi}_{r} & =b(\xi, \eta)+a(\xi, \eta) u \\
\dot{\eta} & =q(\xi, \eta)+p(\xi, \eta) u \\
y & =\xi_{1} .
\end{aligned}
$$

Exact output tracking

$$
y=y_{\mathrm{d}}
$$

requires

$$
\xi=\xi_{\mathrm{d}}:=\left[\begin{array}{lll}
y_{\mathrm{d}}^{(0)} & \cdots & y_{\mathrm{d}}^{(r-1)}
\end{array}\right]^{T}
$$

and

$$
u=\frac{y_{\mathrm{d}}^{(r)}-b\left(\xi_{\mathrm{d}}, \eta\right)}{a\left(\xi_{\mathrm{d}}, \eta\right)}
$$

